\title{
Analysis of Magnetohydrodynamics Flow of Incompressible Fluids over Oscillating Bottom Surface with Heat and Mass Transfer
}

\author{
Vincent M. Bulinda $\mathbb{D}^{\mathbb{D}}$, Giterere P. Kang'ethe, and Phineas R. Kiogora \\ Department of Pure and Applied Mathematics, Jomo Kenyatta University of Agriculture and Technology, \\ Juja 62000-00200, Kenya \\ Correspondence should be addressed to Vincent M. Bulinda; vbulinda@kisiiuniversity.ac.ke
}

Received 24 March 2020; Accepted 13 May 2020; Published 1 June 2020

Academic Editor: Vladimir V. Mityushev

Copyright (c) 2020 Vincent M. Bulinda et al. This is an open access article distributed under the Creative Commons Attribution License, which permits unrestricted use, distribution, and reproduction in any medium, provided the original work is properly cited.

Analysis of magnetohydrodynamics flow of incompressible fluids over an oscillating bottom surface with heat and mass transfer is discussed. The flow is free convection in nature. Momentum, energy, and concentration equations are obtained for computation of their respective profiles. The unsteady flow two-dimensional governing equations are solved numerically by the explicit finite difference method of the Forward Time Backward Space scheme. The numerical results show that the applied parameters have significant effects on the fluid flow and heat transfer and have been discussed with the help of graphical illustrations.

\section{Introduction}

The problems of hydromagnetic free convection flow of incompressible fluids over corrugated vibrating surface have drawn considerable attention from several medical researchers and engineers, resulting in the enhanced heat transfer performance by increasing the area over which heat transfer takes place. This technology is applied in the design of processing equipment that complies with cheap, environmental friendly, and energy-saving with high efficiency of mass and heat transfers. Temperature control is important in corrugated structures manufacturing because it helps to ensure a strong bond between the layers of the corrugated surface and indicates moisture content [1].

Fluid flow and heat transfer on corrugated channel laminar in nature were first studied by [2] for transitional and low Reynolds number turbulent flow. Magnetohydrodynamic free convection flow past an infinite vertical plate oscillating in its plane was studied in the case of an isothermal plate [3]. Rizwan et al. [4] investigated MHD natural convection flow enclosure in a corrugated cavity filled with a porous medium with a complete structure of corrugated surface for heat transfer effects in the presence of the uniform magnetic field. The unsteady flow of second-order thermoviscous incompressible forced oscillations of a fluid bounded by rigid bottom was studied by [5]. Also, Schlichting [6] observed experimentally and numerically that corrugated channels do not have significant effects on heat transfer enhancement if operated in a steady regime. Garg and Maji [7] numerically studied the heat transfer of sinusoidal wavy channels at zero degrees phase shift. A numerical analysis of laminar forced convection in corrugated-plate channels with a sinusoidal, ellipse, and roundedvee wall shapes were studied [8]. Furthermore, Gbadeyan et al. [9] investigated Soret and Dufour effects on heat and mass transfer in chemically reacting $\mathrm{MHD}$ flow through a wavy channel using amplitude as the perturbation parameter.

This work attempts to study the effects of velocities, concentration, and temperature fields on the unsteady flow of incompressible fluid over the heated oscillating bottom for the various material parameters. 


\section{Mathematical Formulation}

Consider a two-dimensional unsteady flow of a viscous incompressible fluid that is electrically conducting flows upwards on an oscillating bottom surface. The $x$-axis is taken along the infinite surface and the $y$-axis normal to it. The corrugated surface and the fluid are initially put at the same temperature. The fluid is initially at rest, responds to the fluctuations of the bottom and the periods of oscillation of the fluid response, and the temperature distribution are assumed to be oscillatory with the same frequency. At time $t>0$, the plate starts vibrating with a frequency of oscillation $\omega$ and reference velocity $U_{R}$ forming boundary oscillating velocity $u=U_{R} \sin \omega t$ approximated [10]. A magnetic field $B_{0}$ of uniform strength is applied perpendicular to the plate along the positive $z$-axis and the magnetic Reynolds number is assumed to be small since the electric intensity $E$ is zero at the plate; therefore, it is assumed to be zero everywhere within the flow [11-13]. The bottom surface is subjected to perturbations through forced transverse oscillations.

Linear sinusoidal displacement of the fluid along the vibrating surface, along the $x$-axis, is generated. Under the Navier-Stokes equation approximation, the viscous stress tensor is zero. The restoring force that produces the oscillation is the buoyancy force, and the waves are associated with these vibrations. The boundary contains the effect of confining the wave energy to a region of finite extent. Corrugation causes periodic variation in the force component and the oscillations due to vortex shedding [14]. Fluid dumping is generated as a result of relative fluid movement to the vibrating structure [15]. It means that the cross-flow vibration is caused by the lift force while the inline flow vibration is caused by the drag force, which in all cases are vortex-induced vibrations represented by periodic corrugation [2]. From Lorentz force, a moving particle with velocity, carrying a charge, contains a force acting on this particle. Also, the magnetic field vector $\mathbf{B}$ is perpendicular to the vibrating surface.

A current is induced in a conducting loop when magnetic flux linking the loop changes [16, 17]. The electric field intensity in a region of time with varying magnetic flux density is present. When the magnetic Reynolds number is small $[3,18]$ the induced magnetic field is negligible in comparison with the applied magnetic field, therefore becoming constant. Since the corrugated bottom surface is nonconducting, therefore the heat flux is zero at the surface and hence zero everywhere in the flow. There is a variation in the temperature and the density, but density is neglected everywhere $[19,20]$ apart from the buoyancy terms which varies linearly with the local temperature and mass fraction.

Under the above-stated conditions and assuming variation of density in the body force term under Boussinesq's approximation [7], the problem is governed by the following momentum equations describing velocity profiles:

$$
\begin{aligned}
\frac{\partial u}{\partial t}+u \frac{\partial u}{\partial x}+v \frac{\partial u}{\partial y} & =v\left(\frac{\partial^{2} u}{\partial x^{2}}+\frac{\partial^{2} u}{\partial y^{2}}\right)+g \beta_{T}\left(T-T_{\infty}\right)+g \beta_{C}\left(C-C_{\infty}\right)-\frac{\sigma u B_{0}^{2}}{\rho} \\
\frac{\partial v}{\partial t}+u \frac{\partial v}{\partial x}+v \frac{\partial v}{\partial y} & =v\left(\frac{\partial^{2} v}{\partial x^{2}}+\frac{\partial^{2} v}{\partial y^{2}}\right)-\frac{\sigma v B_{0}^{2}}{\rho} \\
\frac{\partial C}{\partial t} & =D_{M}\left(\frac{\partial^{2} C}{\partial x^{2}}+\frac{\partial^{2} C}{\partial y^{2}}\right)+\frac{D_{T} k_{T}}{T_{m}}\left(\frac{\partial^{2} T}{\partial x^{2}}+\frac{\partial^{2} T}{\partial y^{2}}\right)-u \frac{\partial C}{\partial x}-v \frac{\partial C}{\partial y} \\
\frac{\partial T}{\partial t}+u \frac{\partial T}{\partial x}+v \frac{\partial T}{\partial y} & =\frac{\kappa}{\rho C_{p}}\left(\frac{\partial^{2} T}{\partial x^{2}}+\frac{\partial^{2} T}{\partial y^{2}}\right)+\frac{\mu}{\rho C_{p}}\left[\left(\frac{\partial u}{\partial x}\right)^{2}+\left(\frac{\partial v}{\partial x}\right)^{2}\right]+\frac{D_{M} k_{T}}{C_{p} C_{s}}\left(\frac{\partial^{2} C}{\partial x^{2}}+\frac{\partial^{2} C}{\partial y^{2}}\right)+\frac{\sigma B_{0}^{2}}{\rho C_{p}}\left(u^{2}+v^{2}\right)+\frac{\partial q_{r}}{\partial z}
\end{aligned}
$$

The heat due to viscous dissipation is taken into an account and thermal radiation is assumed to be present in the form of a unidirectional flux in the $z$-direction denoted by $q_{r}$.

By using the Rosseland approximation [19], the radiative heat flux $q_{r}$ is given by

$$
q_{r}=\frac{4 \sigma_{s}}{3 k_{e}} \frac{\partial T^{4}}{\partial y}
$$

Since temperature differences within the flow are sufficiently small, then (5) can be linearized by expanding $T^{4}$ in Taylor series about $T_{\infty}$, which after neglecting higher-order terms is

$$
T_{\infty}^{4} \cong 4 T T_{\infty}^{3}-3 T_{\infty}^{4} .
$$

Substituting the partial derivative with respect to $T$ of (5) in (4), the rate of change of radiative heat flux becomes

$$
\frac{\partial T}{\partial t}+u \frac{\partial T}{\partial x}+v \frac{\partial T}{\partial y}=\frac{\kappa}{\rho C_{p}}\left(\frac{\partial^{2} T}{\partial x^{2}}+\frac{\partial^{2} T}{\partial y^{2}}\right)+\frac{\mu}{\rho C_{p}}\left[\left(\frac{\partial u}{\partial x}\right)^{2}+\left(\frac{\partial v}{\partial x}\right)^{2}\right]+\frac{D_{M} k_{T}}{C_{p} C_{s}}\left(\frac{\partial^{2} C}{\partial x^{2}}+\frac{\partial^{2} C}{\partial y^{2}}\right)+\frac{\sigma B_{0}^{2}}{\rho C_{p}}\left(u^{2}+v^{2}\right)-\frac{16 \sigma_{s} T_{\infty}^{3}}{3 k_{e}} \frac{\partial^{2} T}{\partial y^{2}} .
$$


With initial and boundary conditions,

$$
\begin{aligned}
& t=0: u=0, v=0, T=0, C=0 \text { at } 0 \leq x \leq L, \\
& t>0: U=U_{\infty}, C=C_{w}, T=T_{w}, C=C_{\infty} \text { at } x=0, \\
& u=U_{R} \sin \omega t, \\
& T=T_{\infty}+\left(T_{w}-T_{\infty}\right), \\
& v=0, \\
& C=C_{\infty}+\left(C_{w}-C_{\infty}\right) \text { at } y=0 .
\end{aligned}
$$

Introducing nondimensional numbers,

$$
\begin{aligned}
x & =L x^{*}, \\
y & =L y^{*}, \\
u & =U_{R} \sin \omega t, \\
v & =U_{R} v^{*}, \\
t & =\frac{t^{*}}{t_{R}}, \\
u & =U_{R} \sin \omega t, \\
v & =U_{R} v^{*}, \\
T^{*} & =\frac{T-T_{\infty}}{T_{w}-T_{\infty}}, \\
U_{R} & =(v g \beta \Delta T)^{1 / 3}, \\
C^{*} & =\frac{C-C_{\infty}}{C_{w}-C_{\infty}}, \\
\Delta T & =T_{w}-T_{\infty}, \\
& \\
U_{\infty} &
\end{aligned}
$$

Equations (1)-(3) and (6) are nondimensionalised as follows.

Momentum equations in $x$-axis and $y$-axis are given as

$$
\begin{aligned}
\frac{\partial u^{*}}{\partial t^{*}}+u^{*} \frac{\partial u^{*}}{\partial x^{*}}+v^{*} \frac{\partial u^{*}}{\partial y^{*}}= & \frac{1}{\operatorname{Re}}\left(\frac{\partial^{2} u^{*}}{\partial x^{* 2}}+\frac{\partial^{2} u^{*}}{\partial y^{* 2}}\right)+\mathrm{Gr}_{\theta} T^{*} \\
& +\mathrm{Gr}_{m} C^{*}-\mathrm{Mu}^{*}
\end{aligned}
$$$$
\frac{\partial v^{*}}{\partial t^{*}}+u^{*} \frac{\partial v^{*}}{\partial x^{*}}+v^{*} \frac{\partial v^{*}}{\partial y^{*}}=\frac{1}{\operatorname{Re}}\left(\frac{\partial^{2} v^{*}}{\partial x^{* 2}}+\frac{\partial^{2} v^{*}}{\partial y^{* 2}}\right)-\mathrm{Mu}^{*} .
$$

The energy equation is given as

$$
\begin{aligned}
\frac{\partial T^{*}}{\partial t^{*}}+u^{*} \frac{\partial T^{*}}{\partial x^{*}}+v^{*} \frac{\partial T^{*}}{\partial y^{*}}= & \frac{1}{\operatorname{Re}}\left[\frac{1}{\operatorname{Pr}}\left(\frac{\partial^{2} T^{*}}{\partial x^{* 2}}+\frac{\partial^{2} T}{\partial y^{* 2}}\right)\right] \\
& +\frac{\operatorname{Ec}}{\operatorname{Re}}\left[\left(\frac{\partial u^{*}}{\partial x^{*}}\right)^{2}+\left(\frac{\partial v^{*}}{\partial x^{*}}\right)^{2}\right] \\
& +D_{f} \operatorname{Re}\left(\frac{\partial^{2} C^{*}}{\partial x^{* 2}}+\frac{\partial^{2} C^{*}}{\partial y^{* 2}}\right) \\
& +\operatorname{Re}\left(u^{* 2}+v^{* 2}\right) \\
& -\left(\frac{4}{3 N \operatorname{PrRe}} \frac{\partial^{2} T^{*}}{\partial x^{* 2}}\right) .
\end{aligned}
$$

The concentration equation is given as

$$
\begin{aligned}
\frac{\partial C^{*}}{\partial t^{*}}+u^{*} \frac{\partial C^{*}}{\partial x^{*}}+v^{*} \frac{\partial C^{*}}{\partial y^{*}}= & \frac{1}{S_{c} R_{e}}\left(\frac{\partial^{2} C^{*}}{\partial x^{* 2}}+\frac{\partial^{2} C^{*}}{\partial y^{* 2}}\right) \\
& +\frac{\operatorname{Sr}}{\operatorname{Re}}\left(\frac{\partial^{2} T^{*}}{\partial x^{* 2}}+\frac{\partial^{2} T^{*}}{\partial y^{* 2}}\right),
\end{aligned}
$$

where

$$
\begin{array}{r}
M=\frac{\sigma B_{0}^{2} u}{\rho U_{R}^{2}}, \\
R_{e}=\frac{U_{R} L}{v},
\end{array}
$$$$
\mathrm{Gr}_{\theta}=\frac{g \beta \Delta T L}{U_{R}^{2}},
$$$$
\mathrm{Gr}_{m}=\frac{g \beta\left(C-C_{\infty}\right) L}{U_{R}^{2}},
$$$$
R_{e}=\frac{U_{R} L}{v},
$$$$
P_{r}=\frac{C_{p} \mu}{\kappa},
$$$$
S_{r}=\frac{\rho D_{M} k(\Delta T)}{\mu T_{m}\left(C_{s}-C_{\infty}\right)},
$$

$D_{f}=\frac{\rho D_{M} k\left(C-C_{\infty}\right)}{\mu c_{s} C_{p}(\Delta T)}$,

$S c=\frac{\mu}{\rho D_{M}}$,

$R=\frac{\sigma B_{0}^{2} \mu}{\rho^{2} C_{p} \Delta T}$

$E c=\frac{U_{R}^{2}}{C_{p} \Delta T}$,

$N=\frac{k_{e} k}{4 \sigma_{s} T_{\infty}^{3}}$. 
With the following initial and boundary conditions,

$$
\begin{aligned}
& t^{*}=0: u^{*}=0, v^{*}=0, T^{*}=0, C^{*}=0 \text { at } 0 \leq x^{*} \leq L, \\
& t^{*}=0: u^{*}=\sin \omega t, v^{*}=0, T^{*}=1, C^{*}=1 \text { at } x^{*}=0, \\
& u^{*}=0, v^{*}=0, T^{*}=T_{\infty}+\left(T_{w}-T_{\infty}\right), \\
& C^{*}=C_{\infty}+\left(C_{w}-C_{\infty}\right) \text { at } y^{*}=0, \\
& u^{*}=0, v^{*}=0, T^{*}=1, C^{*}=1 \text { at } x^{*}=L .
\end{aligned}
$$

The Skin friction, Nusselt number, and Sherwood number at the corrugated surfaces are estimated as follows:

$$
\begin{aligned}
C_{f} & =-\left(\frac{\partial u}{\partial x}\right)_{x=0}, \\
\mathrm{Nu}_{x} & =-\left(\frac{\partial T}{\partial x}\right)_{x=0}, \\
\mathrm{Sh} & =-\left(\frac{\partial C}{\partial x}\right)_{x=0} .
\end{aligned}
$$

\section{Numerical Technique}

The partial differential equations (10)-(14) show the solutions to highly nonlinear coupled governing equations of velocity, concentration, and temperature with the various physical parameters, and the associated boundary conditions in (15) are solved numerically using the explicit finite difference method of the Forward Time Backward Space (FTBS) scheme since this method is stable and is validated using computer software. This is carried out by discretizing the computational domain with nonuniform grids of sinusoidal elements. The flow is in two dimensions and therefore flow domain is confined by the $x, y$, and $t$ axes. The approximate values of $u^{*}, v^{*}, C^{*}$, and $T^{*}$ are found at every nodal point for particular $i$ at $(k+1)$ th time level. A necessary condition for time stability, the Courant-Friedrichs-Lewy (CFL) condition, which depends on time and space discretization, is used. The FTBS finite difference method is applied to replace continuous derivatives with difference formulas that involve only the discrete values associated with positions on the mesh. The basic unknowns for the above differential equations are the velocity components $\left(U_{k, j}^{m+1}, V_{k, j}^{m+1}\right)$, the temperature $\left(T_{k, j}^{m+1}\right)$, and the concentration $\left(C_{k, j}^{m+1}\right)$.

Momentum equations expressed in finite differences is given as

$$
\begin{aligned}
U_{k, j}^{m+1}= & \left(\left[U_{k, j}^{m}-(\Delta t / 2 \Delta x) U_{k, j}^{m}\left(-U_{k-1, j}^{m+1}+U_{k, j}^{m}-U_{k-1, j}^{m}\right)+\left(\Delta t / 2 \operatorname{Re}(\Delta x)^{2}\right)\left(U_{k+1, j}^{m+1}+U_{k-1, j}^{m+1}+U_{k+1, j}^{m}-2 U_{k, j}^{m}+U_{k-1, j}^{m}\right)\right.\right. \\
& +\left(\Delta t / 2 \operatorname{Re}(\Delta y)^{2}\right)\left(U_{k, j+1}^{m+1}+U_{k, j-1}^{m+1}+U_{k, j+1}^{m}-2 U_{k, j}^{m}-U_{k, j-1}^{m}\right)-M(\Delta t / 2) U_{k, j}^{m} \\
& \left.\left.+\left(\Delta t \mathrm{Gr}_{\theta} / 2\right)\left(T_{k, j}^{m+1}+T_{k, j}^{m}\right)+\left(\Delta t \mathrm{Gr}_{m} / 2\right)\left(C_{k, j}^{m+1}+C_{k, j}^{m}\right)\right]\right) \\
& \cdot\left(\left[1+U_{k, j}^{m}(\Delta t / 2 \Delta x)+\left(\Delta t / \operatorname{Re}(\Delta x)^{2}\right)+\left(\Delta t / \operatorname{Re}(\Delta y)^{2}\right)+M \Delta t\right]\right)^{-1}, \\
V_{k, j}^{m+1}= & \left(\left[V_{k, j}^{m}-(\Delta t / 2 \Delta x) V_{k, j}^{m}\left(-V_{k-1, j}^{m+1}+V_{k, j}^{m}-V_{k-1, j}^{m}\right)+\left(\Delta t / 2 \operatorname{Re}(\Delta x)^{2}\right)\left(V_{k+1, j}^{m+1}+V_{k-1, j}^{m+1}+V_{k+1, j}^{m}-2 V_{k, j}^{m}+V_{k-1, j}^{m}\right)\right.\right. \\
& \left.\left.+\left(\Delta t / 2 \operatorname{Re}(\Delta y)^{2}\right)\left(V_{k, j+1}^{m+1}+V_{k, j-1}^{m+1}+V_{k, j+1}^{m}-2 V_{k, j}^{m}+V_{k, j-1}^{m}\right)-M(\Delta t / 2) V_{k, j}^{m}\right]\right) \\
& \cdot\left(\left[1+V_{k, j}^{m}(\Delta t / 2 \Delta x)+\left(\Delta t / \operatorname{Re}(\Delta x)^{2}\right)+\left(\Delta t / \operatorname{Re}(\Delta y)^{2}\right)+M \Delta t\right]\right)^{-1} .
\end{aligned}
$$

The concentration equation expressed in finite differ-

ences is given as

$$
\begin{aligned}
C_{k, j}^{m+1}= & \left(\left[C_{k, j}^{m}-(\Delta t / 2 \Delta x) C_{k, j}^{m}\left(-C_{k-1, j}^{m+1}+C_{k, j}^{m}-C_{k-1, j}^{m}\right)\right.\right. \\
& +\left(\Delta T / 2 \operatorname{ReSc}(\Delta x)^{2}\right)\left(C_{K+1, j}^{m+1}+C_{K-1, j}^{m+1}+C_{K-1, j}^{m+1}+C_{K+1, j}^{m}-2 C_{K, j}^{m}+C_{K-1, j}^{m}\right) \\
& +\left(\Delta t / 2 \operatorname{Re}(\Delta y)^{2}\right)\left(C_{k, j+1}^{m+1}+C_{k, j-1}^{m+1}+C_{k, j+1}^{m+1}-2 C_{k, j}^{m}+C_{k-1, j}^{m}\right) \\
& +\left(\operatorname{sr} \Delta T / 2 \operatorname{Re}(\Delta x)^{2}\right)\left(T_{k+1, j}^{m+1}-2 T_{k, j}^{m+1}+T_{k-1, j}^{m+1}+T_{k+1, j}^{m+1}-2 T_{k, j}^{m+1}+T_{k-1, j}^{m+1}\right) \\
& \left.\left.+\left(\operatorname{sr} \Delta T / 2 \operatorname{Re}(\Delta y)^{2}\right)\left(T_{k, j+1}^{m+1}-2 T_{k, j}^{m+1}+T_{k, j-1}^{m+1}+T_{k, j+1}^{m+1}-2 T_{k, j}^{m+1}+T_{k, j-1}^{m+1}\right)\right]\right) \\
& +\left(\left[1+(\Delta t / 2 \Delta x) T_{k, j}^{m}+\left(\Delta t / \operatorname{ReSc}(\Delta x)^{2}\right)+\left(\Delta t / \operatorname{ReSc}(\Delta y)^{2}\right)\right]\right)^{-1} .
\end{aligned}
$$


The energy equation expressed in finite differences is given as

$$
\begin{aligned}
T_{k, j}^{m+1}= & {\left[\begin{array}{c}
T_{k, j}^{m}-(\Delta t / 2 \Delta x) U_{k, j}^{m}\left(-T_{k-1, j}^{m+1}+T_{k, j}^{m}-T_{k-1, j}^{m}\right)+\left(\operatorname{Df} \Delta T / 2 \operatorname{Re}(\Delta x)^{2}\right)\left(C_{K+1, j}^{m+1}-2 C_{K, j}^{m+1}+C_{K-1, j}^{m+1}+C_{K+1, j}^{m}-2 C_{K, j}^{m}+C_{K-1, j}^{m}\right)+ \\
\left(\operatorname{Df} \Delta T / 2 \operatorname{Re}(\Delta y)^{2}\right)\left(C_{K, j+1}^{m+1}-2 C_{K, j}^{m+1}+C_{K, j-1}^{m+1}+C_{K, j+1}^{m+1}-2 C_{K, j}^{m}+C_{K, j-1}^{m}\right)+\left(\operatorname{Ec} \Delta T / 4 \operatorname{Re}(\Delta x)^{2}\right)\left(U_{k, j}^{m+1}-U_{k, j-1}^{m+1}-U_{k, j}^{m}-U_{k, j-1}^{m+1}\right)^{2}
\end{array}\right] / } \\
& \cdot\left[\begin{array}{c}
1+U_{k, j}^{m}+\Delta t / 2 \operatorname{Repr}(\Delta x)^{2}\left(T_{k+1, j}^{m+1}+T_{k-1, j}^{m+1}+T_{k+1, j}^{m}-2 T_{k, j}^{m}+T_{k+1, j}^{m}\right)+\left(\Delta T / 2 \operatorname{RePr}(\Delta y)^{2}\right)\left(T_{k, j+1}^{m+1}+T_{k, j-1}^{m+1}+T_{k, j+1}^{m}-2 T_{k, j}^{m}+T_{k, j-1}^{m}\right)+ \\
(\Delta t \operatorname{RRe} / 4)\left(\left(U_{k, j}^{m}\right)^{2}+\left(V_{k, j}^{m}\right)^{2}\right)-\left(2 \Delta T / 3 N \operatorname{RePr}(\Delta y)^{2}\right)\left(T_{k, j+1}^{m+1}+T_{k, j-1}^{m+1}+T_{k, j+1}^{m}+2 T_{k, j}^{m}+T_{k, j-1}^{m}\right)
\end{array}\right] \\
& \cdot\left(\left[1+(\Delta T / 2 \Delta x) U_{k, j}^{m}+(\Delta t / 2 \Delta x)+\left(\Delta t / \operatorname{RePr}(\Delta x)^{2}\right)+\left(\Delta t / \operatorname{RePr}(\Delta y)^{2}\right)-\left(4 \Delta T / 3 N \operatorname{RePr}(\Delta y)^{2}\right)\right]\right)^{-1} .
\end{aligned}
$$

To get the analytical results of velocities in (17) and (18), concentration in (19), and temperature in (20) for various values of physical parameters, the code of the algorithm has been executed in MATLAB running on a PC. This is done by applying numerical calculations, and the mesh size is fixed at $\Delta x=0.2, \Delta y=0.3$, and $\Delta t=0.0001$, where a sinusoidal shape is formed by the $x$-axis and $y$-axis containing $15 \times 20$ meshes.

\section{Discussion of the Results}

The effects of various parameters on the flow field of the physical problem for velocities, concentration, and temperature are discussed based on the following considerations:

(i) The value of Prandtl number Pr is taken to be to 0.71 corresponding to air

(ii) The value of Schmidt number $\mathrm{Sc}$ is chosen 0.22 which represents hydrogen at approx. $T_{m}=25^{\circ} \mathrm{C}$ and $1 \mathrm{~atm}$

(iii) The values of Dufour number Df and Soret number $\mathrm{Sr}$ are chosen in such a way that their product is constant provided that the mean temperature $T_{m}$ is kept constant as well

(iv) The radiation parameter $N$ is kept constant at $N=3$ as an indication that there is strong thermal radiation compared with thermal conduction

4.1. Velocity Profiles. The analytical solutions to the coupled equations (10)-(14) together with the boundary condition (15) yield the velocity distribution. Figures 1-10 show the variations of velocity profiles with the different values of different parameters. The graphs shown the figures are generated using MATLAB Code, for instance, by fixing the values of $\mathrm{Gr}_{\theta}=10, \mathrm{Gr}_{m}=10, M=0.2, \mathrm{Ec}=0.5, \mathrm{Df}=0.03$, $\mathrm{Sr}=0.08, \mathrm{Sc}=0.22, R=0.2$, and $\omega t=\pi / 6$ and varying the value $\operatorname{Pr}$.

As shown in Figure 1, the magnitude of the Prandtl number, $\operatorname{Pr}$, is varied in Table 1 , that determines whether the thermal boundary layer is larger for $\operatorname{Pr} \leq 1$, where buoyancy forces are in balance with the thin viscous boundary layer, or smaller for $\operatorname{Pr} \geq 1$, where inertial and buoyancy forces are in balance with the momentum boundary layer; this is shown at the oscillating bottom surface with the wave-like motion. A smaller value of $\mathrm{Pr}$ is an indication that heat diffuses faster than velocity; therefore, it is clear that fluids with small Prandtl numbers are free-flowing liquids with high thermal conductivity and are therefore a good choice for heatconducting liquids, as shown in Figure 1.

Figure 2 shows the effect of Reynolds number on secondary velocity profiles. Since $\mathrm{Re}$ is associated with the smoothness of fluid flow, at lower velocities the flow is laminar and this is pictured as a series of parallel layers moving at different velocities. In the presence of oscillations, the fluid flows vigorously and reaches a velocity at which the velocity changes from laminar to turbulence. When a small Re is used, it applies that the viscous force is predominant thus imposing drug in the fluid and reducing the fluid flow.

The effect of local mass Grashof number $\mathrm{Gr}_{m}$ on velocity is shown in Figure 3. In this case, mass transfer natural convection is as a result of concentration gradients rather than temperature gradients. It is clear that when the value of $\mathrm{Gr}_{m}$ increases, the velocity rises as it reaches the greatest value near the surface due to the enhancement in the buoyancy force (Tables 1-3).

The inline vibration of a structure is caused by the oscillating drag force with different ranges in the reduced velocities. A similar effect is experienced when thermal Grashof number $\mathrm{Gr}_{\theta}$ is used, as shown in Figure 4. By varying the values of $\mathrm{Gr}_{\theta}$, the effects of free convection currents on the flow are indicated and the fluid's velocity increases since fluid flow is aided by the free convection currents.

Figure 4 shows that when the values of $\mathrm{Gr}_{\theta}$ causes a rise in velocity profiles on a cooled surface due to the varying nature of boundary conditions, an indication that the thermal radiation parameter produces significant increases in the thermal conditions of the fluid temperature which consequently induces more fluid in the boundary layer through buoyancy effect to the viscous force, therefore enhancing fluid velocity. Variation in $\mathrm{Gr}_{\theta}$ and $\mathrm{Gr}_{m}$, as shown in Figure 5, has an increasing effect on velocity near the 


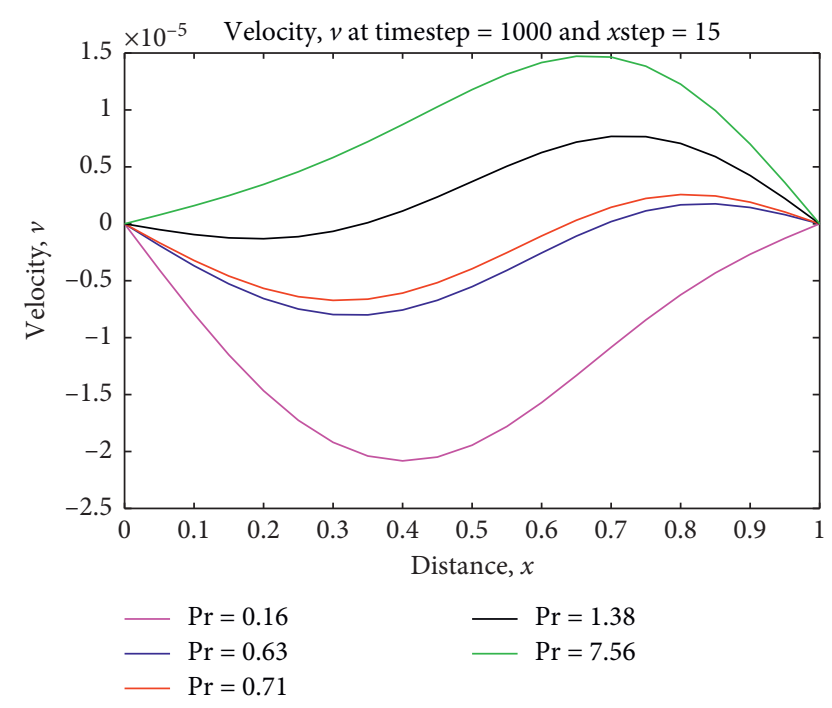

FIGURE 1: Effects of Prandtl number on secondary velocity profile.

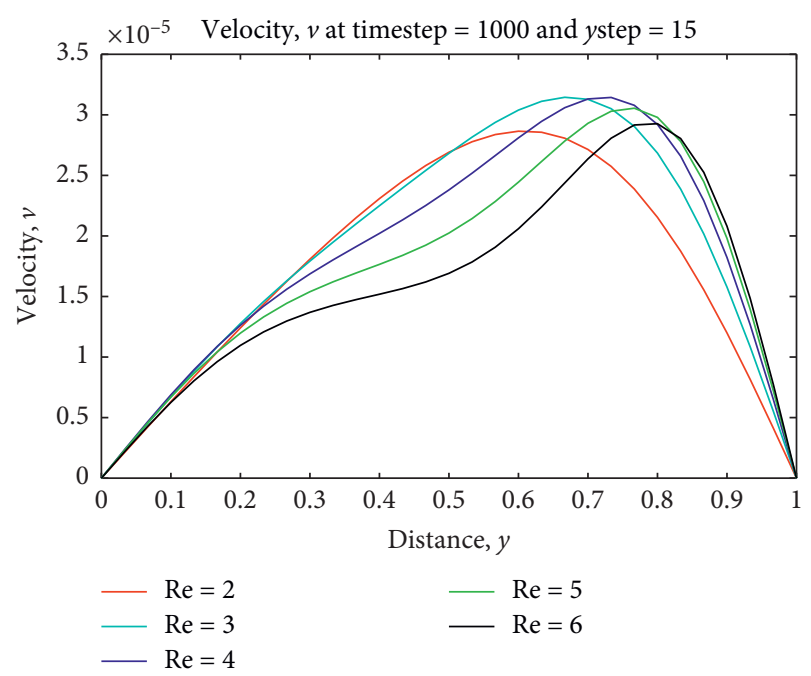

FIGURE 2: Effects of Reynolds number on secondary velocity profile.

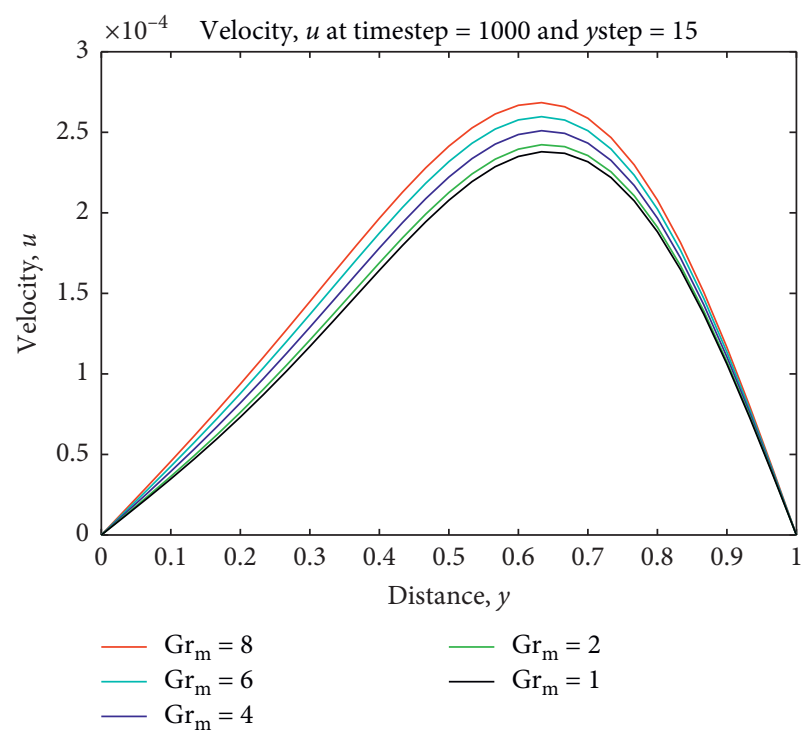

FIGURE 3: Effects of mass Grashof number on primary velocity profile.

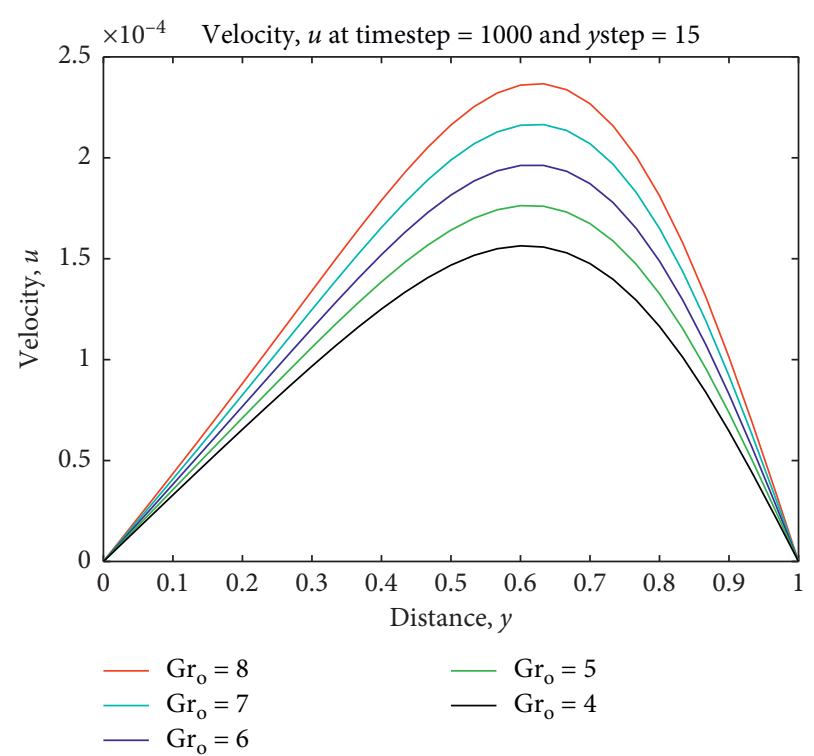

FIGURE 4: Effects of thermal Grashof number on primary velocity profile.

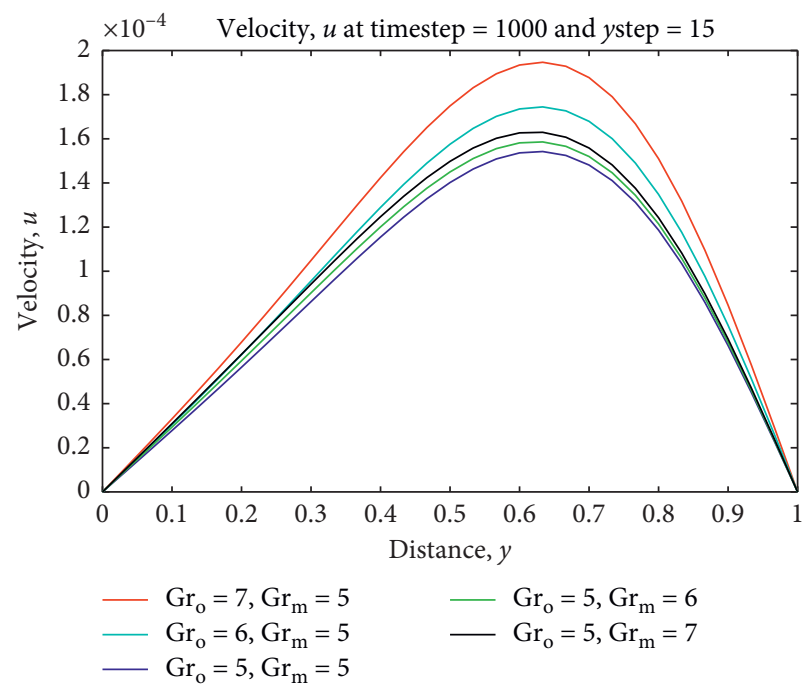

FIGURE 5: Effects of mass and thermal Grashof number on primary velocity profile.

center as a result of thermal and mass buoyancy forces due to cooling of the surface, by making the bond between the fluids to become weaker, strengthening the internal friction to reduce, and the gravity becoming stronger enough. Due to oscillation, thermal and flow patterns adjacent to the boundary are mainly affected.

A reverse effect in the case of heating of the surface, where $\mathrm{Gr}_{m}<0$ and $\mathrm{Gr}_{\theta}<0$, is shown in Figure 6. Reducing the effect on velocity near the center as a result of thermal and mass buoyancy forces due to the heating of the surface is carried out by making the bond between the fluids to become stronger thus strengthening the internal friction to increase and the gravity becoming weaker enough.

It is observed from Figure 7 that while all other participating parameters are held constant, the values of Sc from 


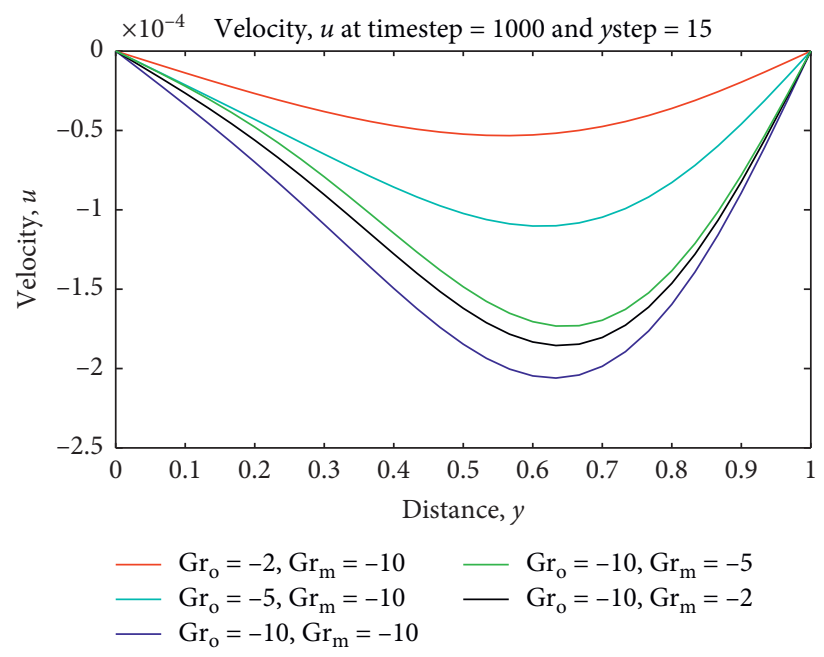

Figure 6: Effects of negative values of mass and thermal Grashof number on primary velocity profile.

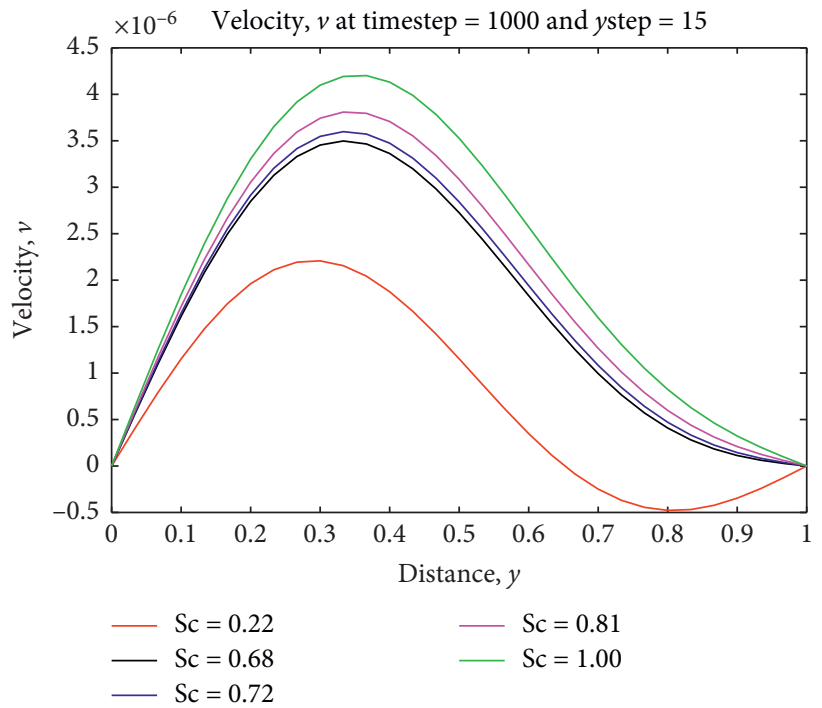

Figure 7: Effects of Schmidt number on secondary velocity profile.

hydrogen to atmosphere pressure reduce the velocity due to oscillation. Since Sc is the ratio velocity boundary layer to the concentration boundary layer which is comparable to Prandtl number in heat transfer, therefore Sc is applied to characterize flows when there are simultaneous momentum and mass transfer.

Figure 8 depicts the effects of velocity on the magnetic parameter $M$. Velocity rises as values of $M$ improves because the frictional or drag force (Lorentz force) in the magnetic field is responsible, which affects the velocity field that opposes the fluid motion, causing the velocity to decrease.

An increase in magnetism significantly reduces the thickness of the boundary layer, thereby reducing the velocity components. A reversal in the direction of the secondary velocity profiles is achieved by using large values of $M$. Here, the effective conductivity of the fluid rises with a rise in $M$ as a result of damping force due to oscillation.
The Soret effect causes the main-flow shear stress to rise and the cross-flow shear stress to fall, as shown in Figure 9. By decreasing the values of $\mathrm{Sr}$ effect leads to a rise in the main flow and cross-flow velocities, as an indication that the velocity boundary layer thickness decreases with an increase in $\mathrm{Sr}$ as a result of mass buoyancy force. This brings about the thermal diffusion effect.

Figure 10 shows that when the values of the Dufour number increases, velocity rises as an indication that the velocity boundary layer thickness increases due to mass diffusion effect.

4.2. Concentration Profiles. Prandtl number shows how fast thermal diffusion takes place in comparison to momentum diffusion. Here, the values of Prandtl number Pr used are $\operatorname{Pr}=0.16$ representing a mixture of noble gases, $\operatorname{Pr}=0.63$ for oxygen, $\operatorname{Pr}=0.71$ for air, $\operatorname{Pr}=1.38$ for gaseous ammonia, 


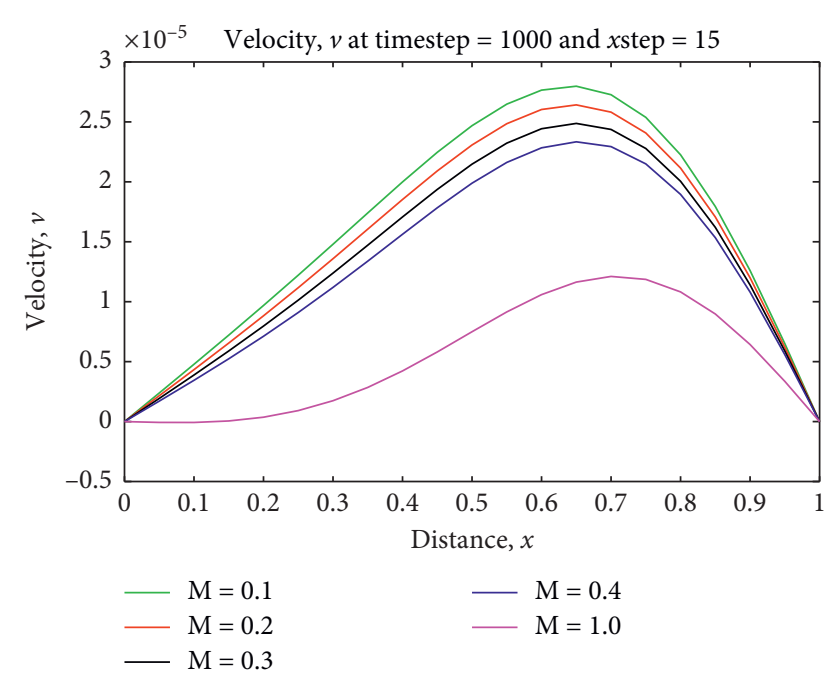

FIGURE 8: Effects of magnetic parameter on secondary velocity profile.

and $\operatorname{Pr}=7.56$ for water at $20^{\circ} \mathrm{C}$ and one-atmosphere pressure. It is clear from Figure 11 that an increase in $\mathrm{Pr}$ causes a fall in concentration due to the Brownian motion of the fluid as a result of an increase in migration from the high concentration regions to the regions with low concentration.

The Reynolds number used is assumed to be small so that the induced magnetic field is neglected within the fluid particles as the fluid moves due to vibration, as shown in Figure 12. It is clear that the Reynolds number is varied in Table 2, to help predict flow patterns in different fluid flow situations.

As the values of Schmidt number rises, i.e., 0.22 (hydrogen), 0.62 (water vapour), and 0.78 (ammonia), from Figure 13, the concentration profile rises because the concentration profile and the boundary layer thickness decreases, corresponding to a thinner concentration boundary layer relative to the momentum boundary layer.

The effect of oscillation on velocity is overcome by freestream velocity, leading to the observed crossover of concentration profiles. When the values of Dufour number increases, the fluid concentration field reduces the boundary layer thickness due to oscillation, as shown in Figure 14.

4.3. Temperature Profiles. An increase in Prandtl number results in a fall in temperature, as shown in Figure 15, because the thermal boundary layer thickness decreases with increasing Pr. This is because the fluid viscosity becomes larger and reduces the thickness of the thermal boundary layer. In cases where Pr is high in liquids, the instability is hydrothermal and the related mechanism involves communication between free-surface temperature perturbations and bulk-liquid temperature. By eliminating the free-surface temperature, oscillations caused by hydrothermal wave coupling could be broken and they would cease. A smaller value of $\operatorname{Pr}$ is an indication that heat diffuses quickly compared to the velocity.

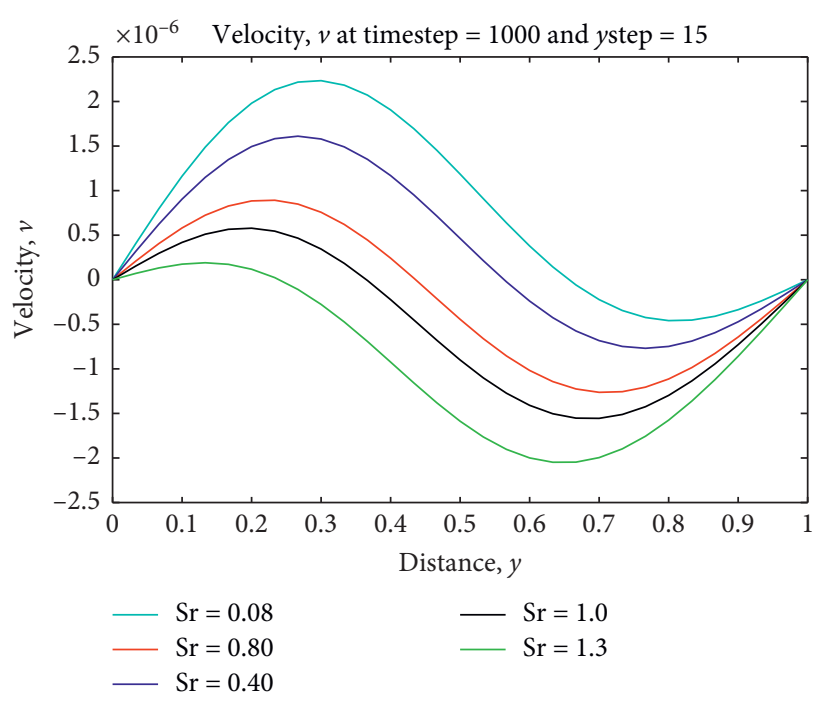

FIGURE 9: Effects of Soret number on secondary velocity profile.

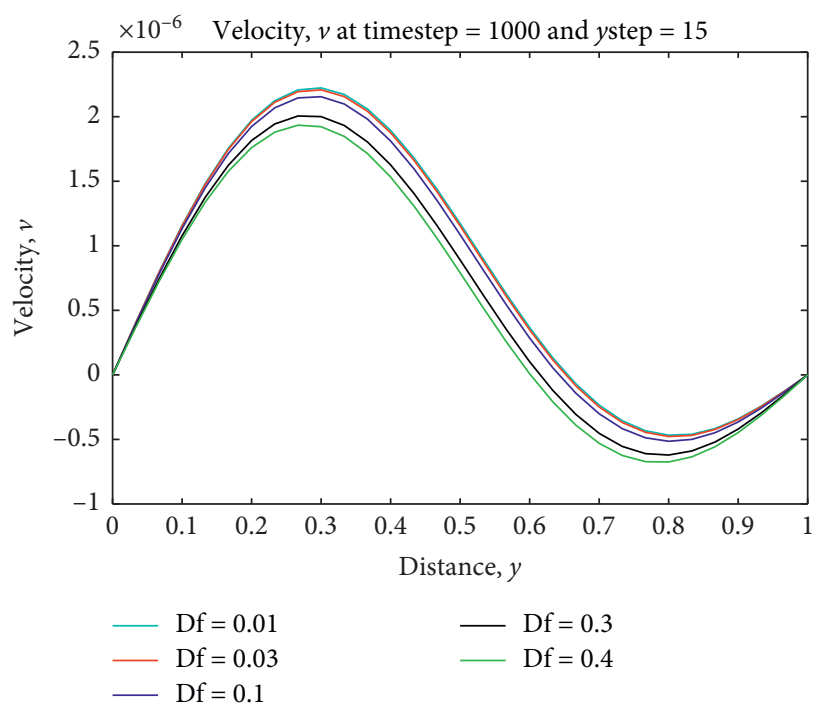

FIGURE 10: Effects of Dufour number on secondary velocity profile.

Reynolds number incorporates the physical properties of liquid density and dynamic viscosity which are directly related to temperature. This means that dynamic viscosity decreases in response to falling density. From Figure 16, as the temperature rises, the change in viscosity decreases due to the presence of the inertia force. Reynolds number is directly proportional to the temperature.

The Eckert number influences the self-heating of a fluid due to dissipation as a result of internal friction of the fluid. If dissipation is neglected at Ec $\leq 1$ as shown in Table 3. Using Figure 17, it is shown that for higher values of the Eckert number Ec, the rate of heat transfer decreases. All the terms in the energy equation describing the effects viscous dissipation and body forces on the energy balance can be neglected, and the equation reduces to a balance between conduction and convection. The effect of viscous dissipation on the flow field is to increase 
TABle 1: Values of the change in Prandtl number.

\begin{tabular}{lcccccccccc}
\hline $\operatorname{Pr}$ & $\mathrm{Re}$ & $\mathrm{Gr}_{\theta}$ & $\mathrm{Gr}_{m}$ & $M$ & $\mathrm{Ec}$ & $\mathrm{Df}$ & $R$ & $\mathrm{Sc}$ & $\omega$ & $\mathrm{Sr}$ \\
\hline 0.16 & 2 & 8 & 10 & 0.2 & 0.5 & 0.03 & 0.2 & 0.22 & $\pi / 6$ & 0.08 \\
0.63 & 2 & 8 & 10 & 0.2 & 0.5 & 0.03 & 0.2 & 0.22 & $\pi / 6$ & 0.08 \\
0.71 & 2 & 8 & 10 & 0.2 & 0.5 & 0.03 & 0.2 & 0.22 & $\pi / 6$ & 0.08 \\
1.38 & 2 & 8 & 10 & 0.2 & 0.5 & 0.03 & 0.2 & 0.22 & $\pi / 6$ & 0.08 \\
7.56 & 2 & 8 & 10 & 0.2 & 0.5 & 0.03 & 0.2 & 0.22 & $\pi / 6$ & 0.08 \\
\hline
\end{tabular}

TABle 2: Values of the change in Reynolds number.

\begin{tabular}{lcccccccccc}
\hline $\operatorname{Pr}$ & $\operatorname{Re}$ & $\mathrm{Gr}_{\theta}$ & $\mathrm{Gr}_{m}$ & $M$ & $\mathrm{Ec}$ & $\mathrm{Df}$ & $R$ & $\mathrm{Sc}$ & $\omega$ & $\mathrm{Sr}$ \\
\hline 0.71 & 2 & 8 & 10 & 0.2 & 0.5 & 0.03 & 0.2 & 0.22 & $\pi / 3$ & 0.08 \\
0.71 & 3 & 8 & 10 & 0.2 & 0.5 & 0.03 & 0.2 & 0.22 & $\pi / 3$ & 0.08 \\
0.71 & 4 & 8 & 10 & 0.2 & 0.5 & 0.03 & 0.2 & 0.22 & $\pi / 3$ & 0.08 \\
0.71 & 5 & 8 & 10 & 0.2 & 0.5 & 0.03 & 0.2 & 0.22 & $\pi / 3$ & 0.08 \\
0.71 & 6 & 8 & 10 & 0.2 & 0.5 & 0.03 & 0.2 & 0.22 & $\pi / 3$ & 0.08 \\
\hline
\end{tabular}

TABLE 3: Values of the change in Eckert number.

\begin{tabular}{lcccccccccc}
\hline $\operatorname{Pr}$ & $\mathrm{Re}$ & $\mathrm{Gr}_{\theta}$ & $\mathrm{Gr}_{m}$ & $M$ & $\mathrm{Ec}$ & $\mathrm{Df}$ & $R$ & $\mathrm{Sc}$ & $\omega$ & $\mathrm{Sr}$ \\
\hline 0.71 & 2 & 8 & 10 & 0.2 & 0.5 & 0.03 & 0.2 & 0.22 & $\pi / 3$ & 0.08 \\
0.71 & 2 & 8 & 10 & 0.2 & 1.0 & 0.03 & 0.2 & 0.22 & $\pi / 3$ & 0.08 \\
0.71 & 2 & 8 & 10 & 0.2 & 2.0 & 0.03 & 0.2 & 0.22 & $\pi / 3$ & 0.08 \\
0.71 & 2 & 8 & 10 & 0.2 & 3.0 & 0.03 & 0.2 & 0.22 & $\pi / 3$ & 0.08 \\
0.71 & 2 & 8 & 10 & 0.2 & 4.0 & 0.03 & 0.2 & 0.22 & $\pi / 3$ & 0.08 \\
\hline
\end{tabular}

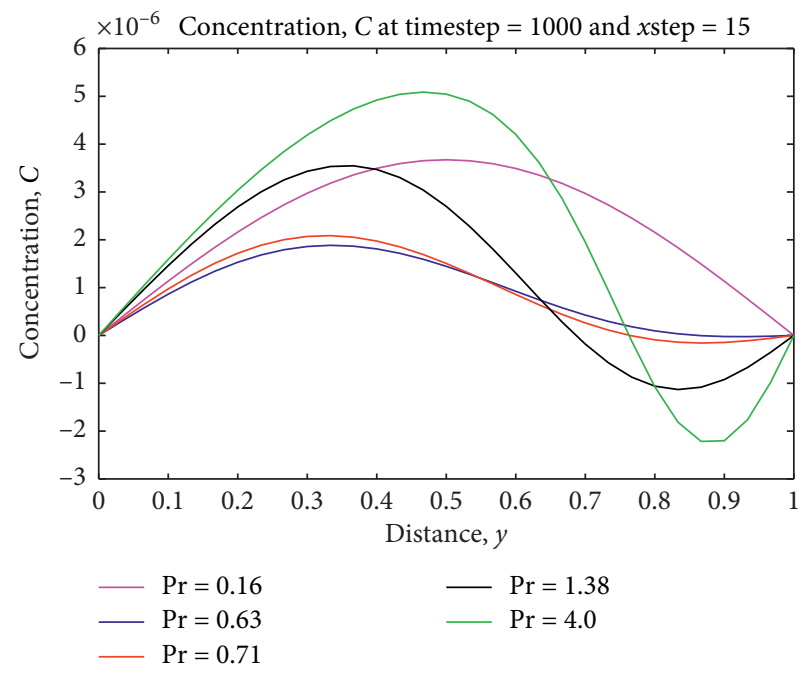

Figure 11: Effects of Prandtl number on concentration profile.

the energy, resulting in greater fluid temperature and as a consequence greater buoyancy force. The increase in the buoyancy force due to an increase in the dissipation parameter enhances the temperature.

Figure 18 shows that the Dufour number is directly proportional to the fluid temperature when other parameters are kept constant as a result of thermal boundary layer thickness.

The values of skin friction, Nusselt number, and Sherwood number are computed from random values generated from MATLAB, as shown in Table 4.

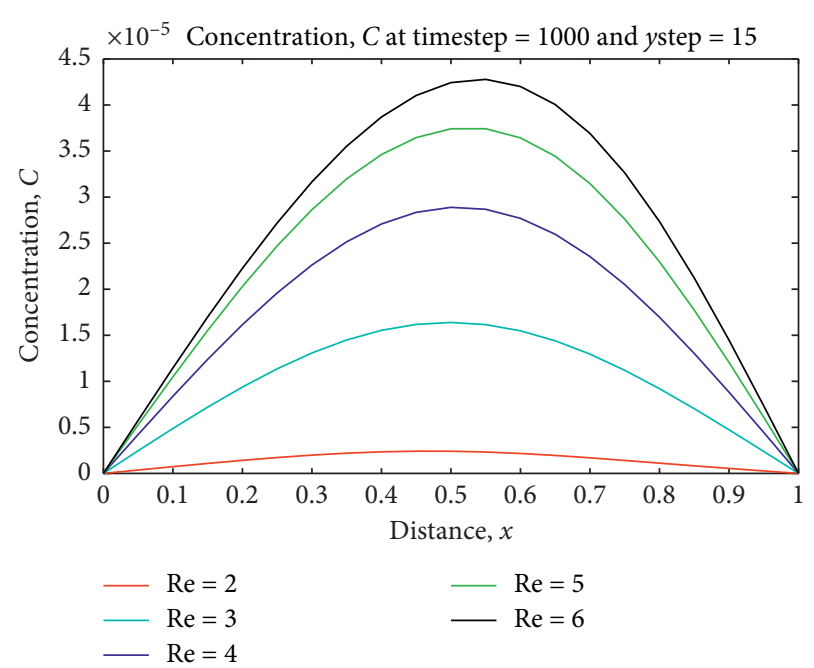

Figure 12: Effects of Reynold number on concentration profile.

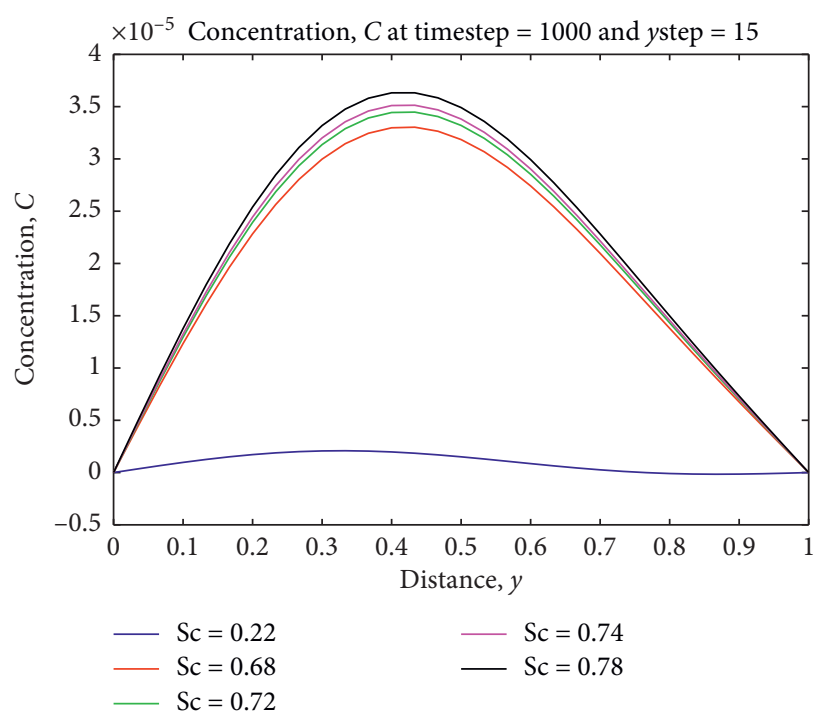

Figure 13: Effects of Schmidt number on concentration profile.

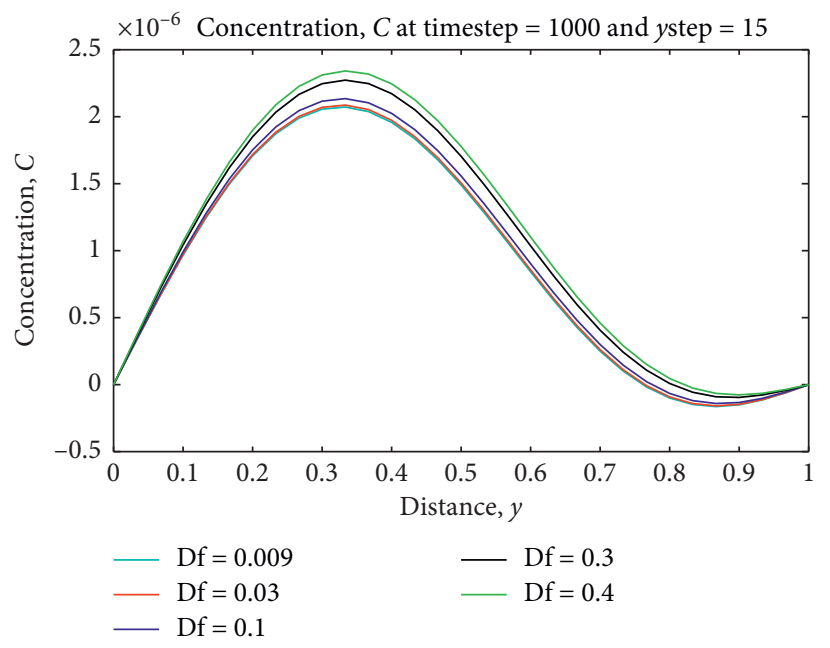

Figure 14: Effects of Dufour number on concentration profile. 


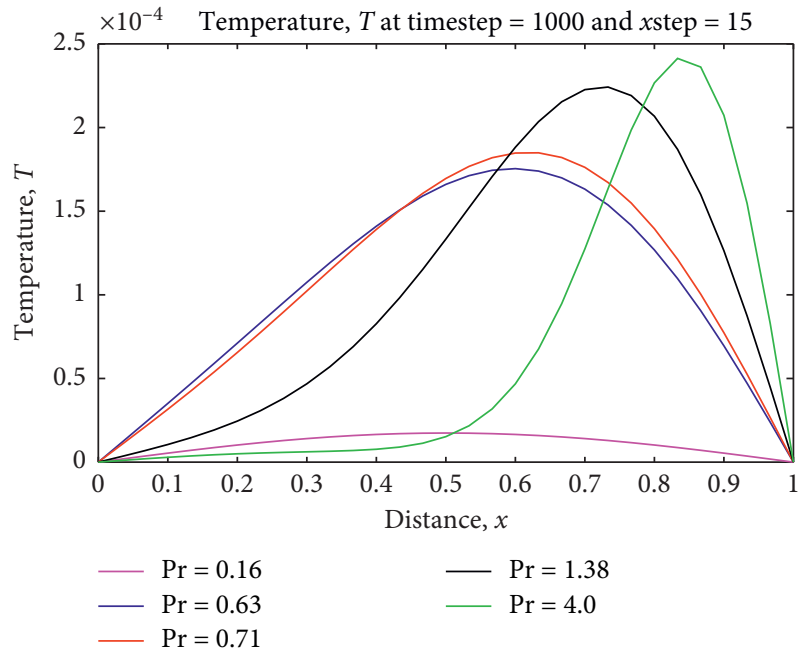

Figure 15: Effects of Prandtl number on temperature profile.

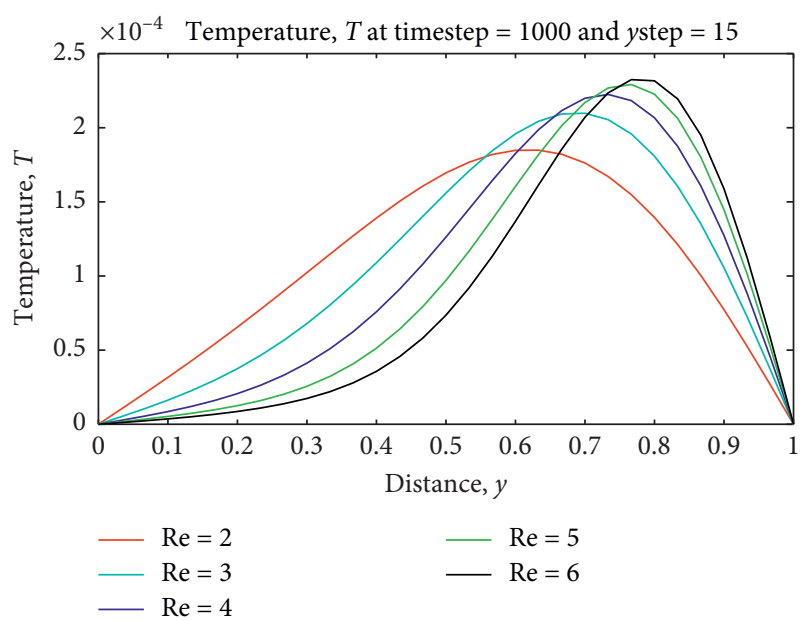

FIGURE 16: Effects of Reynolds number on temperature profile.

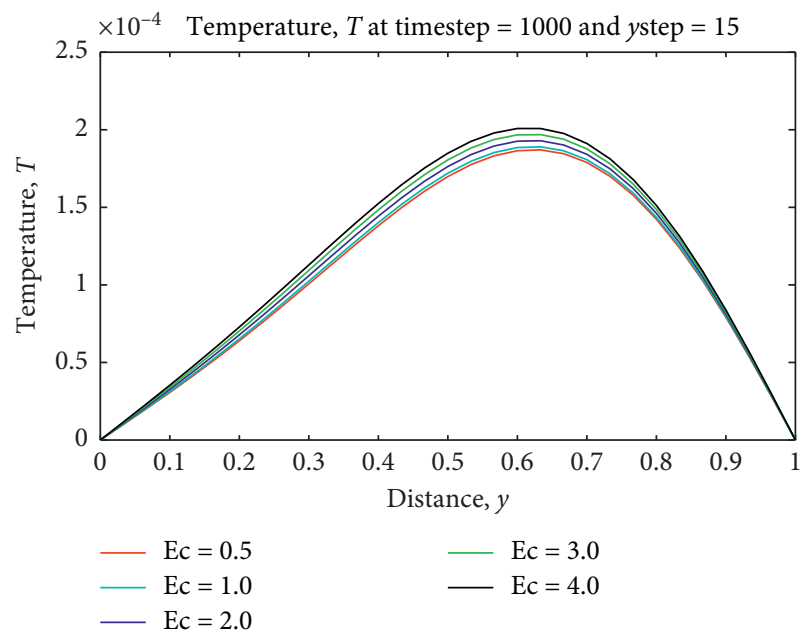

FIgURE 17: Effects of Eckert number on temperature profile.

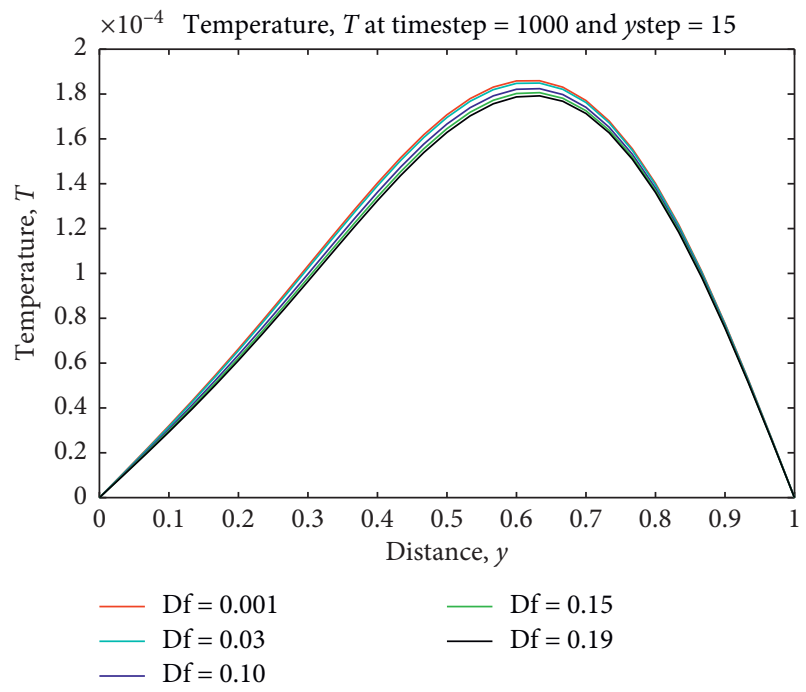

FIgURE 18: Effects of Dufour number on temperature profile.

TABle 4: Values of Nusselt number, Sherwood number, and skin friction.

\begin{tabular}{lccc}
\hline Parameters & $C_{f}$ & $\mathrm{Nu}_{x}$ & $\mathrm{Sh}$ \\
\hline $\mathrm{Gr}_{m}=5$ & 0.4017 & - & - \\
$\mathrm{Gr}_{m}=10$ & 0.3715 & - & - \\
$\mathrm{Gr}_{\theta}=5$ & 0.4132 & - & - \\
$\mathrm{Gr}_{\theta}=10$ & 0.3714 & - & - \\
$\mathrm{Pr}=0.71$ & 0.5501 & -1.3951 & -1.1023 \\
$\mathrm{Pr}=7.0$ & 0.4011 & -5.2947 & -1.0943 \\
$N=3$ & 0.4291 & -4.1803 & -1.0958 \\
$\omega t=0$ & 0.5458 & -2.9984 & -1.1483 \\
$\omega t=30$ & 0.0124 & -2.9983 & -1.1672 \\
$M=0.1$ & 0.0133 & -2.9441 & -1.0841 \\
$M=2.0$ & 0.3097 & -2.9380 & -1.1087 \\
$\mathrm{Sc}=0.22$ & - & -2.9814 & -1.0559 \\
$\mathrm{Sc}=0.68$ & - & -2.9813 & -1.1314 \\
$\mathrm{Sr}=0.08$ & - & -0.8610 & -1.1211 \\
$\mathrm{Sr}=2.0$ & - & -0.8591 & -1.5870 \\
$\mathrm{Ec}=0.5$ & - & -4.7589 & -1.1194 \\
$\mathrm{Ec}=4.0$ & - & -4.0061 & -1.1571 \\
$\mathrm{Df}=0.1$ & - & -2.8015 & -1.1733 \\
$\mathrm{Df}=0.3$ & - & -2.6817 & -1.1764 \\
\hline
\end{tabular}

From Table 4, as time increases, the Nusselt number, Sherwood number, and Skin friction decrease, as it physically implies that shear stresses decrease with an increase in time. A higher value of radiation parameter leads to an increase in magnitudes of skin frictions and Nusselt number as a result of an increase in the rate of species concentration. The effect of radiation is to decrease the rate of energy transport to the fluid, thereby decreasing the temperature of the fluid, but it decreases in Sherwood number.

\section{Conclusion}

A numerical study has been conducted on free convective heat and mass transfer of an incompressible electrically 
conducting fluid on a vibrating bottom surface. Comparing from various pieces of literature, it is clear that

(i) Concentration is directly proportional to Reynolds number and inversely proportional to the Prandtl, Schmidt, Dufour, and Soret numbers, respectively.

(ii) Temperature profiles are directly proportional to the Reynolds, Eckert, and Dufour numbers, respectively, and inversely proportional to the Prandtl number.

(iii) The velocity profiles are directly proportional to Re, $\mathrm{Gr}_{\theta}$, and $\mathrm{Gr}_{m}$ and inversely proportional to $M$.

(iv) Fluid damping is generated as the bottom surface vibrates normal to the flow, and this is proportional to the surface velocity. Therefore, an increase in damping causes an increase in velocity. Therefore, corrugated bottom surface is effective on heat transfer enhancement by breaking and destabilizing the thermal boundary layer.

\section{Nomenclature}

$B_{0}$ : $\quad$ Magnetic field strength $\left(\mathrm{Wbm}^{-1}\right)$

B: $\quad$ Magnetic flux density $\left(\mathrm{Wbm}^{-2}\right)$

Sc: $\quad$ Schmidt number

Sr: $\quad$ Soret number

Df: Dufour number

$D_{M}$ : Molecular diffusion coefficient $\left(\mathrm{m}^{2} \mathrm{~s}^{-1}\right)$

M: $\quad$ Magnetic parameter

$T: \quad$ Dimensional temperature of the fluid (K)

$N$ : $\quad$ Radiative parameter

g: Acceleration due to gravity $\left(\mathrm{ms}^{-2}\right)$

Pr: Prandtl number

Ec: The Eckert number

L: $\quad$ Characteristic length (m)

$\mathrm{Gr}_{\theta}$ : Thermal Grashof number

$\mathrm{Gr}_{m}$ : Local mass Grashof number

$k_{T}$ : Thermal diffusion ratio $\left(\mathrm{m}^{2} \mathrm{~s}^{-1}\right)$

$T_{\infty}$ : Ambient temperature (K)

$T_{m}$ : $\quad$ Mean fluid temperature $(\mathrm{K})$

$T_{s}: \quad$ Wall surface temperature $(\mathrm{K})$

$\mathrm{C}_{\infty}$ : Concentration in the fluid away from the surface $\left(\mathrm{kgm}^{-3}\right)$

$C_{\infty}: \quad$ Concentration at the surface $\left(\mathrm{kgm}^{-3}\right)$

Re: Hydromagnetic Reynolds number

E: $\quad$ Electric field strength $\left(\mathrm{Vm}^{-1}\right)$

M: $\quad$ Magnetic parameter

Sh: $\quad$ Sherwood number

Nu: Nusselt number

Ec: $\quad$ Eckert number

$u, v: \quad$ Dimensionless velocity components

$x, y$ : Cartesian coordinates

$u^{*}, v^{*}$ : Nondimensional velocity components in the $x^{*}$ and $y^{*}$ directions

$R: \quad$ Joules heating parameter

$U_{R}: \quad$ Reference velocity $\left(\mathrm{ms}^{-2}\right)$

q: $\quad$ Velocity vector $\left(\mathrm{ms}^{-2}\right)$

Pr: $\quad$ Prandtl number
$C_{s}: \quad$ Concentration susceptibility parameter $\left(\mathrm{kmolm}^{-3}\right)$

$C_{p}: \quad$ Specific heat at constant pressure $\left(\mathrm{Jkg}^{-1} \mathrm{k}^{-1}\right)$

$k_{e}$ : $\quad$ Mean absorption coefficient $\left(\mathrm{m}^{2} \mathrm{~mol}^{-1}\right)$

$C_{f x}$ : The local skin friction coefficient due the primary velocity profiles

$C_{f y}: \quad$ The local skin friction coefficient due the secondary velocity profiles

$\nabla: \quad$ Gradient operator

Greek Symbols

$\beta$ : Volumetric coefficient of thermal expansion $\left(\mathrm{K}^{-1}\right)$

$\sigma: \quad$ Electrical conductivity $\left(\Omega^{-1} \mathrm{~m}^{-1}\right)$

$\rho:$ Density $\left(\mathrm{kgm}^{-3}\right)$

$\omega t$ : Phase angle

$\sigma_{s}:$ Stefan-Boltzmann Constant $\left(\mathrm{W} \cdot \mathrm{m}^{-2} \cdot \mathrm{K}^{-4}\right)$

$\beta_{T}$ : Thermal expansion coefficient $\left(\mathrm{K}^{-1}\right)$

$\beta_{c}$ : Mass expansion coefficient $\left(\mathrm{K}^{-1}\right)$

$\omega$ : Frequency of oscillation $(\mathrm{Hz})$

$\mu$ : Dynamic viscosity $\left(\mathrm{m}^{2} \mathrm{~s}^{-1}\right)$

$v$ : Kinematic viscosity $\left(\mathrm{m}^{2} \mathrm{~s}^{-1}\right)$

$\alpha$ : Thermal diffusivity $\left(\mathrm{m}^{2} \mathrm{~s}^{-1}\right)$.

\section{Data Availability}

The [MATLAB CODE] data used to support the findings of this study are available from the corresponding author upon request.

\section{Conflicts of Interest}

The authors declare that there are no conflicts of interest regarding the publication of this paper; therefore, the paper can be published after review.

\section{Acknowledgments}

This work was funded by the Kenya National Research Fund (NRF).

\section{References}

[1] N. Pothanna, P. Aparna, and J. Srinivas, "Unsteady forced oscilations of a fluid bounded by rigid bottom," International Journal of Control Theory and Applications, vol. 9, no. 19, pp. 9049-9054, 2016.

[2] L. Goldstein and E. M. Sparrow, "Heat/mass transfer characteristics for flow in a corrugated wall channel," Journal of Heat Transfer, vol. 99, no. 2, pp. 187-195, 1977.

[3] B. Ničene and E. Nobile, "Numerical analysis of fluid flow and heat transfer in periodic wavy channels," International Journal of Heat and Fluid Flow, vol. 22, no. 2, pp. 56-76, 2001.

[4] U. H. Rizwan, M. Toufik, A. S. Feroz, and A. M. Qasem, "MHD natural convection flow enclosure in a corrugated cavity filled with a porous medium," International Journal of Heat and Mass Transfer, vol. 121, pp. 1168-1178, 2018.

[5] N. Pothanna and P. Aparna, "The unsteady flow of a fluid of finite depth with an oscillating bottom," International Frontier Science Letters, vol. 15, pp. 1-8, 2020.

[6] H. Schlichting, Boundary Layer Theory, McGraw-Hill, New York, NY, USA, 7th edition, 1979. 
[7] V. Garg and P. Maji, "Flow and heat-transfer in a sinusoidally curved channel," International Journal of Engineering Fluid Mechanics, vol. 1, pp. 293-319, 1988.

[8] D. M. Ferley and S. J. Ormiston, "Numerical analysis of laminar forced convection in corrugated-plate channels with sinusoidal, ellipse, and rounded-vee wall shapes," Numerical Heat Transfer, Part A: Applications, vol. 63, no. 8, pp. 563-589, 2013.

[9] J. A. Gbadeyan, T. L. Oyekunle, P. F. Fasogbon, and J. U. Abubakar, "Soret and Dufour effects on heat and mass transfer in chemically reacting MHD flow through a wavy channel," Journal of Taibah University of Science, vol. 12, no. 5, pp. 630-651, 2018.

[10] Z. Lei and C. Defu, "Turbulence models for fluid flow and heat transfer between cross- corrugated plates," International Journal of Computation and Methodology, vol. 60, no. 5, pp. 410-440, 2011.

[11] K. Giterere, M. Kinyanjui, and S. M. Uppal, "MHD flow in porous media over a stretching surface in rotating system with heat and mass transfer," International Electronic Journal of Pure and Applied Mathematics, vol. 4, no. 1, pp. 9-32, 2012.

[12] M. Gradeck, B. Hoareau, and M. Lebouché, "Local analysis of heat transfer inside corrugated channel," International Journal of Heat and Mass Transfer, vol. 48, no. 10, pp. 1909-1915, 2005.

[13] I. Nkurikiyimfura, Y. Wang, and Z. Pan, "Heat transfer enhancement by magnetic nanofluids-a review," Renewable and Sustainable Energy Reviews, vol. 21, pp. 548-561, 2013.

[14] T. Nishimura, Y. Ohori, and Y. Kawamura, "Flow characteristics in a channel with symmetric wavy wall for steady flow," Journal of Chemical Engineering of Japan, vol. 17, no. 5, pp. 466-471, 1984.

[15] M. Amielh, F. Anselmet, Y. Jiang et al., "Aeroacoustic source analysis in a corrugated flow pipe using low-frequency mitigation," Journal of Turbulence, vol. 15, no. 10, pp. 650-676, 2014.

[16] N. Ida, Faraday's Law and Induction in Engineering Electromagnetics, Springer, New York, NY, USA, 2000.

[17] J. O'Brien and E. Sparrow, "Corrugated-duct heat transfer, pressure drop and flow visualization," ASME Journal of Heat Transfer, vol. 104, no. 3, pp. 410-416, 1982.

[18] A. S. Idowu, A. Jimoh, and L. O Ahmed, "Impact of heat and mass transfer on MHD oscillatory flow of jeffery fluid in a porous channel with thermal conductivity, Dufour and Soret," Journal of Applied Sciences Environment Management, vol. 19, no. 4, pp. 819-830, 2016.

[19] M. Q. Brewster, Thermal Radiative Transfer and Properties, John Wiley \& Sons, New York, NY, USA, 1992.

[20] R. B. Bird, W. E. Stewart, and E. N. Lightfoot, Transport Phenomena, John Wiley \& Sons, New York, NY, USA, 1966. 